\title{
The Qualitative Analysis of Repertory Grid Data: Interpretive Clustering
}

\section{Abstract 150 words}

This paper presents and illustrates Interpretive Clustering, an innovative and original method of qualitative analysis of Repertory Grid data. Repertory Grids are a popular and flexible method of research, but they have primarily been used to gather data that are analysed quantitatively. Although many researchers have used Grids more qualitatively, this is often limited to a content analysis of the elicited constructs across a sample of participants. Interpretive Clustering is a participant-led method which uses the grid data idiographically to explore how a participant's construing may 'cluster' around one or more issues. We show how this is quite different from a thematic analysis, and discuss how Interpretive Clustering can provide insights that are complementary to those gained from methods like thematic analysis. We conclude with suggestions for how this method, which we argue bridges the qualitative/quantitative divide, might be used in future research.

Keywords: Personal Construct Psychology; Repertory Grid; Interpretive Clustering; thematic analysis 


\section{The Qualitative Analysis of Repertory Grid Data: Interpretive Clustering}

\section{Introduction}

The Repertory Grid was developed by George Kelly (1955) and emerged directly from his Personal Construct Psychology (PCP), both of which are rooted in his clinical psychology practice. Kelly developed the Repertory Grid to enable him to understand his clients' psychological difficulties, and to enable them to gain insight into these, but it has since been used by researchers in a wide variety of topic areas, both within and outside of psychology.

PCP has given rise to a significant body of research methods (see Burr, King and Butt, 2014; Burr, McGrane and King, 2017; Burr, McGrane, Sutcliffe and King, 2019) but the Repertory Grid is by far the most widely known. It is a highly flexible method of data collection that has been adopted in research across a wide range of disciplines, including business, education, tourism and forensic psychology. Repertory grid data consist of a number of constructs (bipolar dimensions of meaning) elicited from and negotiated with the participant (Jankowicz, 2019), along which they place various events, people or objects (referred to as 'elements'), depending upon the topic of study. So, for example, a participant might offer a bipolar construct of 'contented ---- angry' and rate a number of people they know (i.e. elements) as to where they perceive that they fall on this dimension. The bipolar nature of constructs has often led researchers to apply rating scales to them, where the poles of the constructs are placed at opposite ends of the scale and the participant is asked to assign each element a rating on the scale to reflect the personal meaning the elements hold for them.

Repertory Grids enable the researcher to examine what constructs the participant applies to a particular realm of experience and how these constructs are related to each other. But although constructs are essentially qualitative data, an expression of personal meaning, the use of rating scales in Repertory Grids has meant that grids have effectively become predominantly a quantitative method of data collection and have consequently been 
analysed quantitatively, often involving specialist computer software. However, the dangers of reading off psychological meaning from scale data are well known (Jamieson, 2004; Norman, 2010); in particular, scales such as Likert scales are generally treated as producing interval data, but psychologically the 'distance' between scale points may not be equal. Furthermore, applying a 5- or 7-point scale to their constructs may be misleading if participants are only able to make simple discriminations between the two poles. The purpose and utility of the construct was never intended to be measurement, and our qualitative approach moves away from this psychometric development.

Despite the dominance of quantitative analyses of grids, some researchers claim to have used Repertory Grids qualitatively (for example, Hair, Rose and Clark, 2009; Kreber and Klampfeitner, 2013). In actuality, though, this is often limited to a content analysis of the elicited constructs across a number of grids; this is sometimes accompanied by a quantitative analysis of the numerical grid data, but some researchers have developed ways of integrating quantitative and qualitative analyses of grid data, for example, Höft, Heckmann, and Jankowicz (2019).

There are good methodological reasons to consider how the Repertory Grid may be used in a more fully qualitative fashion. Firstly, it sometimes isn't feasible or desirable to use common statistical analyses, such as Principal Component Analysis, in order to examine the relationships between constructs. Bell (2018) argues that such analyses become less reliable when relatively few elements (fewer than 12) are used to elicit the constructs, or where different elements are used with different participants. However, using a smaller number of elements can be methodologically recommended where the aim of the researcher is to explore the participant's construing in depth as part of a qualitative interview. There is also some debate about whether the usual programmes for analysing grids produce psychologically meaningful or trustworthy outputs (ibid.) 
But a second reason concerns what the analysis of the relationships between constructs can offer that is quite different from more familiar forms of qualitative analysis. In the research context, grids are often used as part of an interview with the aim of exploring the dimensions of meaning that a person brings to a particular realm of experience. The most common approach to analysing interview data is some form of thematic analysis (see Braun and Clarke, 2006). Thematic analysis can be applied in a single case or across several cases, with the aim of identifying areas of meaning that share similar features; smaller units of similar meanings are grouped together through 'coding' to constitute higher-order units of sub-themes and, ultimately, over-arching themes. Thematic analysis therefore results in a hierarchical arrangement of themes and sub-themes that, in the view of the researcher, represent key aspects of participants' reported experience.

But an analysis of the relationships between constructs produces something quite different; it identifies the implications that constructs hold for each other. For example, a person may construe others using construct dimensions such as 'self-sacrificing - selfish' and 'lets others walk over them - assertive', but if these constructs are related to each other it may mean that they can have difficulty in becoming more assertive because for them this also implies becoming more 'selfish'. An analysis of the relationships between constructs therefore is not a way of 'theming' constructs, identifying similarities between them, but tells us how the meaning of one construct is informed or inflected by the others to which it is related. Two people may hold apparently similar constructs concerning an issue, and a thematic approach to analysis may place these constructs within the same theme; however, although this theming would suggest that the two individuals perceive that issue in similar ways, the way their constructs are related to other constructs in their individual meaning systems may tell quite a different story. An analysis of the relationships between constructs can therefore complement and enrich the findings from more familiar thematic methods of qualitative analysis. In this paper, we will use our own research to show how grid data may be analysed qualitatively using a method we have termed Interpretive Clustering (IC). We analysed our 
and enrich the findings from a thematic approach.

IC builds on the simple, manual method of establishing relationships between constructs described by Stewart and Stewart (1981). It is a form of clique detection (in particular the socalled maximal clique enumeration problem; see Bron \& Kerbosch, 1973), a clustering method associated with graph and social network theory (Festinger, 1949; Harary \& Ross, 1957). Although yielding identical outputs, IC and the detection of cliques involve different algorithms; IC has the advantage of the availability of a step-by-step manual procedure for cases where a computer program is not available or not desired.

As far as we are aware, clique detection as a method of clustering constructs has not previously been used, and IC is quite different from, and yields different outputs from, commonly used methods for clustering constructs such as hierarchical Cluster Analysis. For example, Jankowicz and Thomas, (1982) provide an account of the FOCUS procedure earlier developed by Shaw and Thomas (see also Stewart and Stewart 1981.)

Hierarchical Cluster Analysis establishes the percentage similarity between pairs of constructs in a grid, and then represents these relationships schematically in a branching 'dendrogram'. In this approach, constructs are assigned to non-overlapping clusters. IC allows the identification of cluster overlaps, where constructs or groups of constructs are 'shared' between different clusters. Furthermore, in Cluster Analysis although the dendogram is produced by the programme, the researcher must then make a judgement as to where to "cut the tree", thereby making a decision about the number of clusters to identify. In Interpretive Clustering the clusters are derived directly from the grid data before interpretation begins, arguably rendering the analysis more faithful to participant meanings. 
Heckmann and Bell (2016) give a short history of the use of clustering in Repertory Grids and also discuss problems associated with this.

$\underline{\text { Other methods for exploring relationships between constructs do exist, for example }}$ 'laddering' (Hinkle, 1965) and 'pyramiding' (Landfield, 1971). These methods are based upon the hierarchical nature of the construct system as conceived by Kelly, with more 'superordinate' constructs subsuming more subordinate ones below them. It is proposed that laddering allows one to 'ascend' the construct system, thereby reaching constructs that are more in the nature of overarching, core values; pyramiding, conversely, descends the hierarchy in order to elicit examples of more concrete, behavioural construing that the participant feels would be 'evidence' of more superordinate constructs or core values. There has been considerable debate as to whether constructs can be usefully understood as arranged in such a hierarchical fashion (see Butt, 1995a, 1995b). However IC, like other forms of construct clustering discussed above, is not aimed at exploring the hierarchical relationships between constructs. Rather, it asks how constructs may share meaning with each other, forming a 'community' that has a distinct character.

The purpose of our research study was to explore the meanings that various kinds of outdoor spaces hold for people. It is well recognised that 'nature' holds a great deal of personal and social significance, but what constitutes 'the natural' for individuals, what it is contrasted with, and the psychosocial meaning of various kinds of outdoor space, can be expected to vary between individuals. We will use findings from our research using Repertory Grids, in which we adopted a thematic content analytic approach as well as IC, showing how these differ and lead to complementary insights. We first explore what meanings about outdoor environments participants held in common and then examine how important individual differences can be revealed lying beneath apparent similarities.

\section{Materials and methods}




\section{$\underline{\text { Recruitment and Participants }}$}

The research was given ethical approval by the University of Huddersfield School of Human and Health Sciences Research Ethics Panel. Participants were recruited opportunistically, but with the intention of obtaining a sample that was reasonably balanced in terms of gender and was as diverse as possible in terms of age, occupation and ethnicity. Thirteen participants were recruited, 8 women and 5 men aged between 22 and 71 . Eleven described themselves as White British, and the other two as mixed Asian and Middle Eastern. Eight were in full-time or part-time employment, 3 were retired and 2 were postgraduate students. All participants were assigned a pseudonym.

\section{Materials}

Eighteen images of outdoor spaces were chosen from a larger pool of 36 obtained from internet sources. Three volunteers each independently sorted the 36 images into three categories: "untamed nature", "managed nature" and "man-made". They then sorted them a second time according to whether they liked, disliked or felt neutral about the images. The final 18 images were chosen with the aim of including six of each environment type, varied in terms of how favourably they were viewed. The degree of consensus amongst the judges was also taken into consideration. Examples of 'untamed nature' included images of jungle, prairie and desert; 'managed nature' included images of agricultural land, a caravan park and commercially grown trees; 'man-made' included images of a children's playground, a quarry and a historic town.

\section{Data collection}

Participants were interviewed individually, and the interviews were audio recorded and later transcribed. Using three images at a time (the 'triadic' method of construct elicitation), participants were asked for any important way in which they saw two as similar to each other and different from the third (Kelly's definition of a construct). For example, they might see two as "unspoiled" in contrast to a third that was "polluted". 'Unspoiled - polluted' is therefore 
a construct. The constructs supplied by each participant were entered into their grid, marking the preferred pole of the construct in each case. The preferred pole is the pole of the construct that the participant would prefer to experience; in the above example the participant might express a preference for "unspoiled" and this would then be their preferred pole.

Participants were then asked to choose, from all the 18 images, seven that represented a selection of those they liked and disliked, and some in between. We considered using all 18 images in the grids, but judged that this would have made the grid completion process very lengthy and tiresome for participants. The actual selection of elements used for each participant was therefore different in each case. This meant that participants' grids were not fully comparable with each other; however, we were not principally interested in making direct comparisons of the construing of particular elements. Having decided on a smaller number of elements, we wanted to ensure that those used in the grid comprised a $\underline{\text { meaningful range for each participant and so took the decision to allow them to choose their }}$ own.

The seven images (elements) they chose were entered at the tops of the columns, and then participants were asked to say, in the case of each of their constructs, which pole each element lay at. Rather than adopting the more usual rating scale as our scoring system, a simple binary scoring system was used, with ticks and crosses representing the two poles of the construct. This simplifies the analysis and enables patterns in construing to emerge clearly. Sometimes, a participant will report that a particular construct cannot meaningfully be applied to an element; in such cases the element is said to be 'out of the range of convenience' of that construct and a score of 0 is entered to indicate this. Each cell of the grid therefore has a tick, a cross or a 0 . An example of a grid, from our participant Jane, can be seen in Fig. 1. 


\section{Data analysis}

Cross-case analysis: content analysis

We used a form of content analysis based on the Core Categorisation procedure described by Jankowicz (2004). The content analysis of constructs can be considered similar to a thematic analysis of conventional interview data in that units of meaning are grouped together under headings decided by the researcher. However, our approach to assigning labels to the resultant categories retained the core feature of constructs as bi-polar. Rather than simply producing theme-type names based on only one pole of the constructs, we developed overarching bi-polar construct names that were judged to represent all the constructs assigned to that category. Although this is more challenging to do than simply giving each category a title as one would for a theme in thematic analysis, it was seen as important to retain the dimensional nature of constructs. For example, both Jane and Pamela used constructs including the pole 'dull'. For Jane the contrast was 'fascinating and stimulating - dull, bland, concrete', but for Pamela it was 'awe-inspiring - dull'. These constructs may not be essentially about the same idea, and so both poles were taken into consideration in categorizing constructs beneath an overarching bi-polar construct.

We applied this method to all the constructs elicited across the sample.

Using 10 of the 13 grids, the second author compiled a list of all constructs, writing each one on a separate piece of card. He then grouped these into themes based on meanings judged to be similar. Where the meaning of a construct was ambiguous or unclear taken out of context, he consulted the participant's transcript; the whole section of the interview in which the construct appeared, sometimes several pages, was read in order to place the construct in a theme appropriate to the participant's meaning. To provide a check on this coding, the first and second authors independently used these themes to code the constructs of the remaining 3 grids, again using the transcripts for guidance where necessary; each construct was either assigned to a theme or coded as 'unsure'. They then compared their 
coding for each construct. Where they were in agreement, the construct was assigned to that theme. Where they were in disagreement or unsure they discussed the construct until agreement was reached. New construct themes were created as necessary. All constructs were then reviewed in the light of the new themes and moved if necessary.

Where the meaning of a construct was ambiguous or unclear, we both consulted the participant's interview transcript was consulted-for context. For example, Hugh supplied the construct 'untamed vs tame, monotonous'. Considering this construct out of context, we were unsure whether it belonged under the overarching construct 'wild, freeform vs order, control, structure' or 'untouched vs human imprint'. We consulted his interview transcript and found that, at the point the construct was elicited, in response to an image of snow-capped mountains, Hugh said:

If people have been up there, they'll have gone up, they'll have found the safest route to get up by and large. So most of it will be, you know, it's about discovery again isn't it, monotony and tame, so if its untamed, you know, it's not entirely untamed, somebody will have found a way up there, but there will be large parts of it where people haven't.

We judged that, for Hugh, this construct was about whether or not people had already established a presence in that environment, so we coded it under 'untouched vs human imprint'

This process produced 15 overarching constructs, plus a small 'miscellaneous' category. There are shown in Table 1, together with the number of constructs assigned to them.

This analysis provided a sense of what meanings the participants held in common. But, as argued above, individual constructs grouped into the same overarching constructs in this way may have subtly different meanings for the 
participants who supplied them, and this was explored through IC to find out whether individual constructs were embedded in rather different systems of meaning for different participants.

Idiographic analysis: Interpretive Clustering

Performing the analysis

Below is an overview of the manual steps involved in IC. A more detailed account of how to perform the analysis manually is provided at https://hhs.hud.ac.uk/InterpretiveClustering/ Alternatively, open access software can be used online at http://ic.openrepgrid.org/

Step 1: Deciding what counts as a match.

The first step is to decide what to accept as an indication that one construct is related to another. As there were seven elements in the present example, we decided that two constructs would count as positively related if they showed the same responses on six out of the seven elements. This is a judgement that the researcher must make and defend; accepting fewer than six 'matches' might render the comparison too near to chance levels, but insisting on seven matches might be so stringent as to eliminate potentially important relationships. 'Matches' can be either two ticks or two crosses (since '0' means that the construct cannot meaningfully be applied to that element, matches of 0 are ignored).

It is also important to recognise that constructs may be negatively related, such that the preferred pole of one construct is related to the non-preferred pole of another. In the case of our research, a negative relationship was 6 or 7 mis-matches between a pair of constructs, again ignoring any 'out of range of convenience' entries.

Step 2: Identifying and counting construct matches.

Each construct is compared with every other construct in the grid, counting for each construct pair the number of elements having the same response, i.e. where the elements 
are placed at the same pole of both constructs. To capture any negative relationships as well as the positive ones, in our research we also noted any construct pairs where there were no matches or only one match (ie. 6 or 7 mis-matches) across the seven elements.

Step 3: Identifying construct clusters.

For each of a participant's constructs, a list of all the other constructs with which it has the requisite number of (either positive or negative) matches is compiled. These lists are inspected to identify all groups of three or more constructs where each construct is related to all the others in the group. To visually depict the clusters and how they may overlap with each other, we have found it useful to prepare a Venn diagram for each participant's clusters. An example of a Venn diagram showing the clusters for our participant Richard can be found in Fig 2. Preferred poles are aligned on the left in each case, and bold font is used to indicate the poles of the constructs that are related to each other.

\section{Comparing individuals}

Inspecting their Venn diagrams tells us a great deal about individual participants and the ways in which they are similar or different to each other in their construing. Our participants differ in terms of how many clusters were identified for them, the extent to which these overlap with each other, and the number of constructs that appear in the clusters. These features tell us about the complexity or simplicity of the person's construing and the extent to which their meaning-making around a topic is ultimately focussed on a single issue, a narrow range of issues, or a much broader range of issues.

For example, Amina's grid contained 13 constructs, but her analysis resulted in two clusters containing only six of these (see Fig. 3), suggesting that she construes the environment in a wide range of ways that do not necessarily cohere or revolve around key issues. Her clusters focus on 'openness' which implies enjoyment, interest and freedom, and also a sense of emptiness where time passes slowly. By contrast, 16 of Diane's 19 constructs are 
found in her 5 clusters (see Fig.4). These constructs revolve around a clear distinction between the human and the natural world that appears to make these two realms separate for her- the wild and natural, which she values, seems incompatible with the presence of human life, which suggests that nature has been 'tampered with'. Almost her entire system of meaning is arranged around this issue. Some constructs therefore seem to be focal points in a participant's construing, drawing other constructs around them and giving them particular inflections of meaning.

The clusters also tell us about the extent to which the person's meanings are related to each other and implicate one another; in terms of the current research, this can be expressed with the phrase "If I think of an environment as having this quality, then it must also have that quality too". For example, in Richard's Venn diagram we can see that 'changeable' also implies physically challenging' and 'a new experience'.

In cases where a content analysis of constructs has been carried out, as in our research, further insight into the subtle differences between individuals can be gained by comparing participants whose clusters contain constructs from the same category theme. Below, we compare the clusters of four participants, Hugh, Jane, Sylvia and Amina. The clusters of all four participants contained one or more constructs from the category "Novelty, excitement, interest - Familiar, dull". This construct theme concerns environments that are construed as stimulating, interesting and arousing of curiosity, being varied or unfamiliar; the contrast is environments that are seen as boring, bland and either uniform or too familiar. But by examining the way these constructs relate to others in their clusters we can see that their meaning is subtly different in the case of each participant. The constructs in this category that appeared in these participants' clusters are shown in Fig. 5. Venn diagrams for Hugh, Jane and Sylvia can be found in Figs. 6-8. 
From an inspection of their Venn diagrams, we can see that the four participants differ markedly in the complexity of their clusters. Amina's is the least complex arrangement, with two clusters which overlap by sharing a single construct. Both Hugh and Sylvia have rather similar, and more complex, arrangements of clusters; both have three clusters, where each cluster shares one or more constructs with one other cluster. Jane's clusters are more complex still; she has six clusters, and each of them overlaps with up to three others and 14 of Jane's 17 constructs are represented in her clusters, suggesting a lot of interconnectedness in her meaning-making. All of Jane's clusters contain at least one construct from the overarching construct category "Novelty, excitement, interest - Familiar, dull" (6 'novelty' constructs in all), suggesting that this is a key concern for her; it suffuses all her construing about the environment. For Sylvia, Amina and Hugh, their one or two constructs in this category appear in only one of their clusters in each case, suggesting that this is not an especially key concern for them.

The implications of their 'novelty' constructs appear to some degree similar for Sylvia and Hugh. For both of these participants, their 'novelty' constructs are related to that which is 'dramatic'; however, the meaning of 'dramatic' (the preferred pole for both Hugh and Sylvia), as defined by what they are contrasted with, is somewhat different. For Hugh, the contrast is 'safe' but for Sylvia it is 'unvarying, goes on and on', and her cluster includes the construct 'variable - doesn't change' and 'verdant - dead, nothing living', emphasising the importance of life, and the novelty of variety and change for her. When looking at an image of prairie grasslands, she recalled a visit to Canada: "It's very unvarying, you can feel it will go on and on and on and on, like the Canadian prairies." She contrasted this with an image of mountains, which she saw as 'dramatic'. Later in her interview, when viewing an image of high-rise flats she described them as "relentless" and said: "It's unvarying because it's extremely efficient to pile people up in little stacks like that... I think that's oppressively unchanging, it's oppressive, that." 
For Hugh, the 'dramatic' is unfamiliar, inspires a sense of wonder and discovery, and is not 'safe'. Hugh's 'novelty' constructs are also clustered with 'natural, chaotic, unpredictable ordered'; we sense in these clusters the thrill that he gains from being in environments that are challenging and offer opportunities for discovery. In his interview, he said:

I love places where you can sort of get lost in your imagination thinking "How would I get up there and how would I get down, what am I going to find when I do get up there?"

A little later, in response to an image of farmlands with distant hills, he said:

It does feel like there's places to explore. I sort of wonder what's behind each rise and fall and what's behind that hill there... but I suppose, the appealing thing about this is its quite a different environment to what I'm used to, just by the fact that its different, its appealing, I don't recall seeing that anywhere I've been before.

Like Hugh, Jane's clusters also feature the issue of safety. In fact her clusters include several constructs showing a concern for lack of safety and her anxiety that she may be unable to cope with such environments and the fear they induce in her, so whereas safety is the non-preferred pole for Hugh, it is something of a pre-occupation and concern for Jane. One or more of her 6 'novelty' constructs appear in every one her clusters, so this issue is seemingly also at the heart of her construing. However, they are clustered with 'safety' constructs in a way that suggests the desires for both novelty and safety are in tension with each other. For example, the constructs 'fascinating, stimulating - dull, bland, concrete', 'arouses curiosity - formulaic' and 'adventurous, a challenge - familiar' share a cluster with 'peaceful - adrenaline-raising' and 'can easily negotiate - unsafe', where 'peaceful' and 'can easily negotiate' are preferred poles. It seems that she is drawn towards environments that are fascinating and arouse her curiosity (her preferred poles), but these are likely to also be 
adrenaline-raising and unsafe (her non-preferred poles). In her interview, she comments on an image of a jungle, and seems to suggest that 'adventure' and 'challenge' are, by their very nature, 'unsafe' and therefore incompatible:

In the sense that that would be not familiar that would be, you know, adventurous, that would be you know, a challenge...and probably not safe, for me, I would think...but then that's similar to adventurous and a challenge. I mean if it was safe, it wouldn't be, would it?

A similar tension can be noted in Sylvia's clusters. While she expressed a consistent preference for wildness, freedom and expansiveness, in one of her clusters this was in tension with her construct 'cosy - chaotic'; the wild, free and expansive is related to 'chaotic', but her preferred pole is 'cosy'. Follow-up interviews could potentially explore these tensions.

For Amina, there is a close relationship between (absence of) boredom, and freedom, openness and enjoyment. Open spaces give a sense of freedom and provide opportunities for enjoyment, and are therefore 'not boring'. When comparing images of high-rise flats, a muddy field and a prairie, she aligns lack of boredom with the freedom to run around:

These two [the prairie and muddy field], I mean this [the prairie] is not too boring, I would say that this [the flats] is boring. I live in a place like this, before, so it's, you know, it's a small space and lots of concrete and ... I feel like it's boring, less freedom... and this one, here [the muddy field? prairie?], this, I think these are weeds or some kind of growing, so it's more like I feel like I'm more free, that I, you know, obviously you can run here [the muddy field? prairie?], you can see the nature. Here [the muddy field] it's not that bad, you know, I mean of course it looks muddy. Compared to here [the flats], it's not that boring, 
Later in her interview, she compares images of a quarry, a caravan park and arable land:

Yeah, there's no enjoyment here. I mean there can be some here [the caravan park], I mean obviously this is people going there with the aim of, you know, camping and so probably getting more enjoyment and this [the arable land] is again work, but I guess when people are, like I remember my grandparents, they are, they have their own space and they're not farming, they're just for themselves, so it can be, when I go there, I see them dealing with the soil and planting things can be, but they're not that stressed. But people working in that type of environment [the quarry] probably not enjoying their life, at least that's my assumption, maybe they are.

Enjoying oneself in the outdoors, having freedom and space seem to define what is 'not boring' for Amina, so 'novelty' carries rather different meanings for her compared to the other participants.

IC arguably provides, in the case of each of our four participants, a nuanced understanding of the implications of 'novelty' for them. They desire novelty, the unfamiliar and unconventional but for each of them this carries different connotations and implications.

\section{Discussion}

We argue that IC represents an original contribution to the qualitative analysis of Repertory Grid data, and has significance for research in the wide range of topics for which Repertory Grids are often adopted. The examination of overlapping construct clusters renders the analysis thorough, and the process of analysis itself, whether conducted manually or with the aid of computer software, is methodologically rigorous.

\section{Our thematic analysis of constructs was a useful first step in identifying the key issues that} were important across our participant group in relation to outdoor spaces. Through using IC, 
we were then able to 'drill down' into the system of meaning of each individual, showing how apparently similar concerns can have subtly different implications for different individuals.

\section{The combination of these two methods allowed for both nomothetic and idiographic analyses}

\section{of the same data set, enabling us to identify both what aspects of their construing}

participants shared as well as what was unique to them. As an idiographic analysis, IC can

therefore usefully complement a more traditional thematic approach to the analysis of qualitative data, providing a more enriched and nuanced understanding of the data; this in turn is likely to prompt new avenues of enquiry and raise fresh research questions. For example, in our research the issue of 'safety' and its sometimes complex relationship to nature, wildness and adventure is one that deserves further investigation, and may not have emerged in quite the same way based only on a thematic approach to our data analysis.

Furthermore, we believe that IC may be especially fruitful in applied research which can lead to interventions or changes in practice. For example, the benefits to health and wellbeing of nature-based interventions such as walking in natural environments are now widely accepted (Capaldi et al., 2014; Chawla, 2015; Mitchell, 2013; Olafsdottir et al., 2017). However, it seems likely that these benefits may depend upon the particular and personal meanings that 'nature' holds for individuals. In our research, we found that the concept of 'freedom' was important to several participants, but for some this implied the enjoyment of being in wide, open spaces and for others it implied a lack of boundaries and restrictions. Interventions could therefore be tailored to individual needs.

Another example is the field of Inter-professional working. Using the 'Pictor' method, which has its origins in PCP theory, King et al (2017) found that personal relationships were deemed important by professionals working as part of multi-professional teams. However, it seems likely that the notion of 'personal relationships' has different meanings and implications for different individuals; for example, it may mean 'friendliness' to one person 
and 'professionalism' to another, with different implications for practice. IC could potentially be used to explore differences in these meanings between individuals.

\section{A participant-led analysis}

Research using a PCP framework aims to help people to reflect upon and gain insight into their own construing; the focus is upon allowing the perspective of the participant to emerge, the interpretation of the data should be participant- rather than researcher-led.

In most other forms of qualitative analysis, such as thematic analysis, the researcher is urged to remain 'close to the data' in the early stages of coding; the aim is to remain faithful to the participant's meaning and not over-interpret the data. However, the analysis is inevitably researcher-led, since interpretation begins relatively early in and throughout the analysis process. Interpretive Clustering provides a way of qualitatively analysing Repertory Grid data that delays the 'moment' of interpretation until late in the analysis process, after the clusters have been generated; the interpretation thereby remains as faithful as possible to participants' own elicited meanings (see Jankowicz, 2019 for a discussion of the challenges of this in Repertory Grid method).

The analysis of the four cases we have presented was undoubtedly an interpretative process, but interpretation was the final stage at the end of a standardised, routinized analytic process. In completing the Repertory Grid, participants are implicitly communicating to the researcher the relationships between their constructs, even though they may struggle to articulate this if asked. By deriving construct clusters from the data, the researcher is arguably remaining faithful to the structure of the participant's construing and delaying their own interpretation until the final stage of analysis.

\section{$\underline{\text { Interpretive Clustering and Thematic Analysis }}$}


IC does not perform the same function as a thematic analysis; theming and clustering are not different ways of achieving the same analytic ends, as illustrated by the two forms of analysis we have presented above. Researchers therefore need to be clear about what kind of analysis will suit their needs. A thematic analysis aims to make sense of a data set (either several cases or a single case) by drawing out similarities and differences. By contrast, IC identifies the implications that constructs hold for each other. For example, for Richard the closely related constructs of 'natural - built environment", 'unspoilt - developed' and 'freedom - constraint' do not constitute three different instances of the same idea or experience, as in a theme. Rather, 'natural' also implies 'unspoilt' and 'freedom'; if an environment is construed by Richard as 'natural' it is also likely to be seen as 'unspoilt' and offering 'freedom'.

\section{Blurring the boundaries between qualitative and quantitative methods}

Since IC involves frequency counts it undeniably has a quantitative component.

Furthermore, the use of computation to derive clusters may at first glance seem to locate IC as a quantitative method. However, the emphasis is clearly upon the in-depth exploration of personal meaning, not upon establishing trends across a data set, and this is the domain of qualitative research. We therefore argue that IC serves to question the division between qualitative and quantitative methods. It is of course not the first method to do so; $Q$ Methodology, for example, has long been regarded as combining the strengths of both types of methods (Sell and Brown, 1984; Brown, 1996).

\section{$\underline{\text { Challenges and limitations }}$}

All methods have limitations as well as strengths, and the researcher must judge whether, for their research purposes, the latter outweigh the former. Like other qualitative methods, the data derived from IC does not represent a complete picture of a person's meaning-

\section{making. Not only is the outcome influenced by the particular elements chosen for the grid}

\section{(like the questions chosen for an interview), the clusters resulting from IC do not capture}


everything that is of significant in a person's construing. For example, a single construct may

$\underline{\text { be of high importance in understanding the meaning of a place (or event or person) to an }}$

$\underline{\text { individual and yet bee unrelated to other constructs. Our decision to use only seven }}$

elements in the grids increased the likelihood that some elicited constructs would not be

perceived as applicable to some of the images, thereby increasing the number of scores of 0

in the completed grids. However, even after eliminating these from our analysis, the high degree of interrelatedness between constructs in many of the grids was still apparent.

Like most qualitative methods, Interpretive Clustering can be time-consuming, especially if undertaken manually. Collecting the Repertory Grid data can also be intensive; in our research, the interviews took between one and two hours each, but this is arguably comparable to 'normal' semi-structured interviews.

But we argue that PCP methods such as construct elicitation have advantages; they allow the researcher to quickly focus on particularly relevant and meaningful aspects of the participant's experience, creating data that is both rich and economical. Although audio recording and transcribing grid interviews is advisable, it is not usually necessary to fully analyse the interviews, which are mainly used to elaborate on the construct analysis.

The Repertory Grid method can also appear rather formulaic. It can look like a standardised and routinized approach, but actually requires as much researcher skill and flexibility as other qualitative methods. When eliciting constructs, the researcher must employ 'active listening', making continual judgements about which elements to present to the participant in order to probe into interesting areas of their construing. They must also feel confident to probe participants when the construct they have just supplied seems to need 'unpacking', and be able to help participants find the words to express their construing without leading them. 
With their strong privileging of the participant's perspective, and their ability to provide rich idiographic analysis, we argue that PCP methods such as Interpretive Clustering constitute useful additions to the qualitative researcher's repertoire.

\section{Acknowledgements}

We are indebted to Prof Devi Jankowicz for his help and advice in preparing this paper.

\section{References}

Bell, R 2018, 'Why do we use repertory grids?' Paper presented at the XIVth Biennial Conference of the European Personal Construct Association, Edinburgh.

Braun, V and Clarke, V 2006, 'Using thematic analysis in psychology', Qualitative Research in Psychology, vol 3, pp. 77-101.

Bron, C and Kerbosch, J 1973, 'Algorithm 457: Finding All Cliques of an Undirected Graph', Communications of the ACM, vol 16(9), pp. 575577.

https://doi.org/10.1145/362342.362367

Brown, S R, 1996, 'Q Methodology and Qualitative Research', Qualitative Health Research, vol. 6(4), pp. 561-567. https://doi.org/10.1177/104973239600600408

Burr, V, McGrane, A and King, N 2017, 'Personal Construct qualitative methods', in P Liamputtong (ed.) Handbook of Research Methods in Health Social Sciences, Springer Verlag, Singapore, DOI https://doi.org/10.1007/978-981-10-2779-6_23-1.

Burr, V, McGrane, A, Sutcliffe, J and King, N 2019, 'Personal Construct Psychology methods for qualitative research', in P Atkinson, S Delamont, A Cernat, JW Sakshaug \& RA Williams 
(eds.) in Sage Research Methods Foundations, SAGE Publications Ltd, London, doi: $10.4135 / 9781526421036865522$.

\section{Butt, TW 1995a, The ordinal relationship between constructs, Journal of Constructivist}

\section{Psychology, vol. 8, pp. 227-236}

\section{Butt, TW 1995b, What is wrong with laddering?, Changes, vol. 13, pp. 82-87.}

Capaldi, CA, Dopko, RL and Zelenski, JM 2014, 'The relationship between nature connectedness and happiness: a meta-analysis', Frontiers in Psychology, vol. 5, https://doi.org/10.3389/fpsyg.2014.00976

Chawla, L 2015, 'Benefits of nature contact for children', Journal of Planning Literature, vol. 30(4), pp. 433-452.

Festinger, L 1949, 'The analysis of sociograms using matrix algebra, Human Relations, vol. 2(2), pp. 153-158, https://doi.org/10.1177/001872674900200205

Hair, N, Rose, S and Clark, M 2009, 'Using qualitative repertory grid techniques to explore perceptions of business-to-business online customer experience', Journal of Customer Behaviour, vol. 8(1), 51-65.

Harary, F, and Ross, I C 1957, 'A procedure for clique detection using the group matrix, Sociometry, vol. 20(3), pp. 205-215, https://doi.org/10.2307/2785673

Heckmann, M and Bell, R C 2016, 'A new development to aid interpretation of hierarchical cluster analysis of repertory grid data', Journal of Constructivist Psychology, vol. 29(4), pp. 368-381, https://doi.org/10.1080/10720537.2015.1134368 


\section{Hinkle, DN 1965, The change of personal constructs from the viewpoint of a theory of}

\section{implications. Unpublished PhD thesis, Ohio State University, Columbus.}

Höft, N, Heckmann, M. and Jankowicz, D 2019, 'Systematic integration of quantitative measures into the qualitative content analysis of constructs', Journal of Constructivist Psychology, vol. 32(4), pp. 345-369, DOI: 10.1080/10720537.2018.1515044

Jamieson, S 2004, 'Likert scales: how to (ab)use them', Medical Education, vol. 38(12), pp. 1217-1218. doi:10.1111/j.1365-2929.2004.02012.x

Jankowicz, D 2004, The Easy Guide to Repertory Grids, Wiley, Chichester.

Jankowicz, D 2019, 'Sociality as negotiation in the research grid interview', Personal Construct Theory and Practice, vol.16, pp.94-99.

Jankowicz, D and Thomas, L 1982, 'An algorithm for the cluster analysis of Repertory Grids in Human Resource development', Personnel Review, vol. 11 (4), pp. 15-22.

Kelly, GA 1955, The Psychology of Personal Constructs, Norton, New York.

King, N, Bravington, A, Brooks, J, Melvin, J and Wilde, D 2017, "'Go make your face known": collaborative working through the lens of personal relationships', International Journal of Integrated Care, vol.17 (4): 3, pp1-11.

Kreber, C and Klampfeitner, M 2013, 'Lecturers' and students' conceptions of authenticity in teaching and actual teacher actions and attributes students perceive as helpful', Higher Education, vol. 66, pp. 463-487. 


\section{Chicago.}

Mitchell, R 2013, 'Is physical activity in natural environments better for mental health than physical activity in other environments?', Social Science and Medicine, vol. 91, pp. 130-134.

Norman, G 2010, 'Likert scales, levels of measurement and the "laws" of statistics', Advances in Health Sciences Education, vol. 15, pp. 625-632.

https://doi.org/10.1007/s10459-010-9222-y

Olafsdottir, G, Cloke, P and Vögele, C 2017, 'Place, green exercise and stress: an exploration of lived experience and restorative effects', Health and Place, vol. 46, pp. 358365.

Sell, DK, and Brown, SR, 1984, 'Q methodology as a bridge between qualitative and quantitative research: Application to the analysis of attitude change in foreign study program participants', In JL Vacca and HA Johnson (eds.) Qualitative Research in Education (Graduate School of Education Monograph Series), Kent State University, Bureau of Educational Research and Service, Kent, OH, pp. 79-87.

Smith, J and Osborn, M 2003, 'Interpretive phenomenological analysis', In JA Smith (ed.) Qualitative Psychology: a practical guide to research methods, Sage, London, pp.51-80.

Stewart, V and Stewart, A 1981, Business Applications of Repertory Grid, McGraw-Hill, London. 
Table 1 Overarching constructs and numbers of constructs assigned to them.

\begin{tabular}{|c|c|}
\hline Overarching construct & N. of constituent constructs \\
\hline Novelty, excitement, interest - Familiar dull & 24 \\
\hline Untouched - Human imprint & 23 \\
\hline Safe, controllable - Dangerous, Threatening & 17 \\
\hline Wild, free form, unconstructed - Order, control, structure & 17 \\
\hline Grand scale, sense of freedom in scale - Small scale, closed & 16 \\
\hline Quiet, peaceful - Busy, activity & 14 \\
\hline Life sustaining - Barren, un-sustaining & 12 \\
\hline Friendliness, belonging - Loneliness, impressiveness & 7 \\
\hline Enjoyment, fun - Functional, not enjoyable & 6 \\
\hline Positive view of how land is used - Negative view of how land is used & 6 \\
\hline Agreeable temperature - Disagreeable temperature & 6 \\
\hline Vibrant, change, movement - Static, unchanging & 6 \\
\hline Energising clarity - stressful & 6 \\
\hline Visually attractive - Visually unattractive & 4 \\
\hline Historic - Recent & 2 \\
\hline Miscellaneous & 2 \\
\hline
\end{tabular}




\section{Figure captions}

Fig 1 Jane's grid

Fig 2 Richard's clusters

Fig 3 Amina's clusters

Fig 4 Diane's clusters

Fig 5 Constructs in the category Novelty, excitement, interest - familiar, dull found within the clusters for Hugh, Sylvia Amina and Jane

Fig 6 Hugh's clusters

Fig 7 Sylvia's clusters

Fig 8 Jane's clusters 